\title{
MedienPädagogik
}

www. medienpaed.com

Zeitschrift für

Theorie und Praxis

der Medienbildung

ISSN 1424-3636

Themenheft Nr. 23: Visuelle Kompetenz

\section{Fotografiegeschichte greifbar machen - Ein Modell transmedialer Vermittlung}

Mandy Gnägi

\begin{abstract}
Mit dem Modell transmedialer Vermittlung wird ein Unterrichtskonzept vorgestellt, das auf mehrsemestrigen Erfahrungen an verschiedenen Fachhochschulen der Schweiz beruht. Die methodische und pädagogische Versiertheit der Dozierenden einerseits und die unterschiedlichen Kompetenzen der Studierenden andererseits nutzend, führt das Modell aus einem vermeintlichen Chaos der heutigen Bilderflut heraus und schafft gemeinsam mit den Studierenden eine geordnete Übersicht für die Vermittlung von Fotografiegeschichte. In dem Modell kommen sowohl neue Medien zum Tragen, mit denen die Studierenden als mehrheitlich «digital natives» unterrichtsspezifische Inhalte in interne und externe Blogs, Bild- und Filmdatenbanken, Diskussionsforen, u. a.m., übertragen, als dass dies auch explizit mit der Arbeit an einer Zeitleiste verknüpft wird. Die Studierenden bringen darauf in regelmässigen, diskursiv und reflexiv aufgebauten Unterrichtseinheiten gedruckte Bilder und auf Notizzettel notierte Schlagworte zur Geschichte und Theorie der Fotografie sowie der allgemeinen Kulturgeschichte an. Durch diese transmedial angelegte Vermittlung sind Studierende Lehrende und Lernende gleichermassen und bilden auf dieser Grundlage ihre visuelle Kompetenz aus, machen Zusammenhänge sowie Beziehungen sichtbar und Fotografiegeschichte greifbar.
\end{abstract}

Nichts ist schwerer zu wissen, als was wir eigentlich sehen.

(Merleau-Ponty 1966, 82)

Wenn Studierende verschiedener Fachklassen für Fotografie aufgefordert werden, wichtige Positionen aus der Geschichte ihres Faches, relevante Namen, entscheidende Techniken oder bildliche Erzählstrategien zu notieren, sind die Ergebnisse meist überschaubar. ${ }^{1}$ Sie sind als mehrheitlich «digital natives»² (Prensky 2001, 1;

1 Dieser Artikel beruht auf mehrsemestrigen Unterrichtserfahrungen an verschiedenen Fachhochschulen der Deutschschweiz.

2 Unter dem von Marc Prensky geprägten Begriff versteht dieser «all «native speakers» of the digital language of computers, video games and the Internet», die im Gegensatz zu den «digital immigrants [...] an outdated language» $(2001,1,2)$ sprechen. In einer neuen Studie aus dem Jahr 2012 wird dies erneut ausgelotet und insbesondere unter den Kategorien «Generationen, Altersspezifik und Nutzung von Medien» (Ganguin u. Meister 2012, 12) sowohl theoretisch als auch empirisch diskutiert. 
Ganguin u. Meister 2012) zu diesem Zeitpunkt noch nicht in der Lage, die sie umgebende Bilderflut einer post-«picturial turn»-Ära ${ }^{3}$ zu kanalisieren und entsprechend einzuordnen. Viele Studierende geben als Ziel ihres Studiums gleichwohl an, selbst Geschichte schreibende Fotografien zu realisieren. Sie möchten Menschen durch ihre Werke berühren und dabei ästhetische, ethische, politische, kurz: gesellschaftsrelevante Positionen markieren.

Angesichts dieser visuellen Inkompetenz (Huber 2004a, 16) einerseits und dem weit reichenden Anspruch andererseits stellt sich die Frage, wie ein fotografiehistorischer Unterricht zur Ausbildung einer visuellen Kompetenz aussehen könnte. Welche Chancen liegen in der Nutzung der multimedial zur Verfügung stehenden Bilder? Kann der bereits seit den 1960er Jahren monierten mangelnden Reflexion hinsichtlich der massenkulturellen Entstehung und Distribution von Bildern (exemplarisch Adorno 1970) ${ }^{4}$ und damit einer unzureichend ausgebildeten Medienkompetenz (Huber 2004a, 26-27) durch eine Stärkung der visuellen Kompetenz innerhalb des fotografiehistorischen Unterrichts entgegen gewirkt werden? Wie können komplexe Bezüge und Zusammenhänge aufgezeigt und ausserdem der auch subjektiv vorhandene warburgsche Bilderatlas jeder Studentin und jedes Studenten - virtuell auf eigenen Datenbanken, öffentlichen Plattformen und Foren, imaginär (Macho 1999; Sowa u. Uhlig 2006) sowie konkret in Form von Fotoalben und Abzügen - fruchtbar gemacht werden? Können mit dem darzulegenden Modell transmedialer Vermittlung Methoden aufgezeigt werden, die visuelle Kompetenz der Studierenden innerhalb oft weniger und nur temporär durchgeführter Lektionen zu stärken und dabei ein realistisches Mass an fotografiehistorischen Inhalten zu vermitteln?

\section{Fotografiehistorischer Unterricht und visuelle Kompetenz}

«Kompetenz ist die Zuschreibung einer bestimmten Eigenschaft an eine Person durch einen Beobachter zweiter Ordnung» (Huber 2004b, 31) konstatiert der Kunsthistoriker Hans Dieter Huber und gibt zu bedenken, dass es sich dabei nicht um fest definierbare Eigenschaften bestimmter Fähigkeiten handle, "sondern um einen sozialen Beobachtungs-, Bewertungs- und Zuschreibungsprozess.» Zur Beobachtung, Beurteilung und Messung visueller Kompetenz schlägt Huber aus diesem Grund den Begriff der Performativität vor, der das mögliche Produkt der handelnden Person sowie einen sichtbaren Prozess umfasst. Während Kompetenzen also nicht sichtbare Fähigkeiten, Kenntnisse und ein eben solches Wissen be-

3 Hierunter versteht William John Thomas Mitchell «rather a postlinguistic, postsemiotic rediscovery of the picture as a complex interplay between visuality, apparatus, institutions, discourse, bodies, and figurality [...] the realization that spectatorship may be as deep a problem as various forms of reading [...] and "visual literacy» might not be fully explicable on the model of textuality.» (Mitchell 1994, 16).

4 Anders als in diesen Kritiken der 1960er Jahre hat die Forschung auch die Chancen des strukturellen und medialen Wandels erkannt. Vgl. dazu z. B. Schulze 2003. 
schreiben (Huber 2004a, 21-23; 2004b, 33; Zawicki-Richter, Bäcker u. Hanft 2010, 2), zeige sich im sichtbaren Handeln und Darstellen der performative Grad (Posner 2003, 20-21; Schaper 2010, 30). Das Curriculum hat sich an Fachhochschulen weitestgehend den veränderten Berufsfeldern angepasst; die Ausbildung zum Bildermachen geht Hand in Hand mit der Ausbildung des Sehens und Wahrnehmens (Weingarten 1999; Sowa u. Uhlig 2006, 78), der Verortung von Kunstwerken im Kontext ihrer Zeit, um dann daraus Schlüsse zu ziehen und Erkenntnisse zu formulieren. Aber «das Sehen setzt das Denken voraus, das wiederum als Basis der Wahrnehmung fungiert», (Bering 2002, 89-90) resümiert der Kunsthistoriker Kunibert Bering zu Gotthold Ephraim Lessings klassische Ausführungen zum Laokoon und dem konstatierten "Zusammenhang zwischen der optischen Wahrnehmung und dem Denken» sowie ihrer «Interdependenz». Visuelle Kompetenz müsse daher durch mehr als nur ein Fach innerhalb eines Curriculums gelehrt werden, um «die pure Konsumentenhaltung ebenso wie jeden Eskapismus» zu überwinden und «die Nutzung der Medien» sowie bildkonstitutiver Welten als Kommunikationsmöglichkeiten zu erkennen. ${ }^{5}$ Der einstige Fokus einer technikorientierten Ausbildung und der damit verbundenen Ausformung einer Bildkompetenz hat sich zu einer möglichst umfassenden Wahrnehmung der Umwelt verlagert (Schaper 2010, 32-33).

Hinsichtlich der angehenden Fotografinnen und Fotografen bedeutet dies, die historische wie gegenwärtige Relevanz angewandter und künstlerischer Fotografien heraus zu arbeiten. Gerade in der komparatistischen Analyse wird der Kanon einer Bildfindung deutlich, der sich aus einer vorwiegend europäischen Bildtradition speist (Bering 2002, 91). Das relevante Bild- und Informationsmaterial ist, und das ist der auszunutzende Vorteil fotografiehistorischen Unterrichtens an Fachhochschulen, durch Lehrende und Lernende gemeinsam zu beschaffen. Je nach den jeweiligen Interessensbereichen der einzelnen Personen sind diverse Medien synergetisch zu nutzen und bereichern durch ihre Disparität wiederum die inhaltliche Ausrichtung des Unterrichts (Helmke 2009, 68).

Der nachfolgend vorzustellende Vorschlag einer transmedialen Vermittlung zielt auch darauf ab, der Theorie visueller Kompetenz ein auf Theorie gestütztes praxiserprobtes Modell an die Seite zu stellen, um damit sowohl die Diskussionen über visuelle Kompetenz einerseits als auch über visuelle Performativität andererseits zu ergänzen. ${ }^{6}$ Die Studierenden erleben angeleitet, aber im eigenen Handeln die

5 Als weitere Elemente für die Ausbildung einer visuellen Kompetenz führt Kunibert Bering die Erlangung von Medienkompetenz an, Wissen und Analysetechniken aus den Bereichen Kunst- und Architekturgeschichte sowie -theorie, Kenntnis historischer Zusammenhänge und Gestaltungsmittel sowie der Positionierung des Ichs (Bering 2002, 90-93).

6 Die Betonung des verwendeten Begriffs Modell verweist auf die Wandelbarkeit der dahinter liegenden Struktur, denn die Beobachter zweiter Ordnung verfügen über eine intersubjektive Beobachterkompetenz, die entsprechend unterschiedlich gewichteter Aspekte visuelle Kompetenz ab- oder zusprechen (Huber 2004b, 36-37). 
mediale Breite, inhaltliche Tiefe, inter- und transmediale Verknüpfung von Fotografie als Teil ihrer Kulturgeschichte. Offensiv wird in diesem Modell der Wahl der Studierenden Rechnung getragen, nämlich sich für eine praktische Ausbildung auf Fachhochschulebene entschieden zu haben. Erfahrungsgemäss sind sie dem historisch ausgerichteten, zumal theoretischen Unterricht in der Regel weniger offen gegenüber eingestellt. Aussagen wie «lch bin kein Theoretiker», «Die Texte der Wissenschaftler verstehe ich ohnehin nicht», "Was interessieren mich die alten Meister, ich bin Fotografin», bis zu «Geschichte habe ich gehasst» begegnen nicht selten und werden den Dozierenden gewissermassen als Haltung einer self-fulfilling prophecy demonstriert. Die Aussagen spiegeln ferner durchaus die eingangs skizzierten Erfahrungen zu Beginn des fotografiehistorischen Unterrichts wider. Doch genau dieselben Studierenden waren am Ende des Semesters in der Lage, bestimmte Fotografien auf Grund ihrer Technik und Ästhetik, des abgebildeten Sujets und des erworbenen Fachwissens, in die entsprechende Zeit einzuordnen sowie Bezüge zur Kunst- und Kulturgeschichte herzustellen. Der reflektierende Umgang bescheinigt damit die von Huber geforderte Performativität als ein in die Öffentlichkeit getragener Ausdruck einer intersubjektiven, visuellen Kompetenz.

\section{Handlungskontext}

Wird die aktuelle Studie des kanadischen Professors für Erziehungswissenschaften, John Hattie, über Lernprozesse und Steigerung der Lerneffektivität auf den Handlungskontext an Fachhochschulen übertragen, ergibt sich eine grundlegende Schwierigkeit, die weniger bei den Studierenden zu suchen ist, als vielmehr bei einer Mehrzahl der Dozierenden. ${ }^{7}$ Denn den Studierenden stehen selten Dozierende gegenüber, die über eine pädagogische Ausbildung verfügen. Als fachliche Expertinnen und Experten sind sie gegenüber einem spezifischen Bildungsauftrag verpflichtet, bei dessen Erfüllung offensichtlich davon ausgegangen wird, dass das Gegenüber unanhängig von Didaktik und Methodik des Unterrichtens, den Inhalt aufzunehmen, anzuwenden und umzusetzen in der Lage ist. Eine der Haupterkenntnisse von Hatties «Synthesis of over 800 meta-analyses relating to achievement» für die Nachhaltigkeit des Unterrichtens ist die Forderung nach Lehrpersonen, die vielfältige Möglichkeiten des Lernens innerhalb des Unterrichts bieten sollten, falls Lernende Sachverhalte wie Aufgaben nicht verstehen oder bewerkstelligen können (Hattie 2009, 22-24). Diese Forderung ist nicht neu und liess Kunibert Bering im Hinblick auf die Erlangung visueller Kompetenz einige Jahre vor Hattie entschieden erklären: «Ein visuell kompetentes Individuum entsteht al-

7 In den von John Hattie $(2009,2011)$ für diesen Artikel herangezogenen Büchern geht es ganz dezidiert darum, wie Lernprozesse sichtbar gemacht und Lerneffizienz steigernd in den Unterricht an graduate schools eingebracht werden können. Diese Form der Performativität hat motiviert, im Folgenden nach Rahmenbedingungen, Möglichkeiten sowie Lehrkompetenzen der Dozierenden an Fachhochschulen zu fragen und methodische Vorschläge zur Diskussion zu stellen. 
lerdings nicht ex nihilo, vielmehr ist entschiedene pädagogische Zuwendung notwendig, um diesen Prozess der Identitätserhaltung und der Orientierung erfolgreich durchzuführen.» ${ }^{8}(2002,91)$ Über eine derartige Lehrkompetenz verfügt aber unter Umständen die Fachperson nicht. Vielmehr wird bereits bei Erstsemestern vorausgesetzt, dass sie über Lernkompetenz verfügen und in der Lage sind, diese bei dem Besuch von Vorlesungen und Seminaren anzuwenden. Der Alltag sieht jedoch anders aus. Denn wissenschaftliches Arbeiten mit der Handhabung entsprechender Techniken - vom Finden von Literatur bis zum exzerpierenden Lesen, vom Verfassen eines lesbaren Textes bis zur Kenntlichmachung verwendeter Bücher, von der Fähigkeit, eigene Gedanken auf der Basis eines Kanons zu formulieren bis zum Halten von Referaten sowie von der Reflexion des Gelernten und der Übertragung auf den Untersuchungsgegenstand - gehört nicht in den Kompetenzbereich der Studierenden, da das primäre schulische Bildungsziel an Lernergebnissen orientiert ist. Franz Emanuel Weinert zur Folge hätten Studierende in der Tat das Gefühl, nicht für das Lernen vorbereitet zu sein, womit letztlich eine wesentliche Voraussetzung für effizientes Lernen an Fachhochschulen nicht erfüllt ist (Weinert 2009, 46).9 Fachhochschüler verfügen aber über mediale Handlungsräume, die aktiv in den Unterricht mit einbezogen werden können: Sie haben in der Regel tragbare oder stationäre Computer mit entsprechender Ausstattung, nutzen das Internet auf vielfältige Weise, besitzen mindestens eine eigene Fotokamera und bewegen sich in Kunst- und Fotografieausstellungen. Somit kann die Frage nach der Ausbildung visueller Kompetenz durch einen möglichst breit angelegten und lustvollen Unterricht unter Nutzung aller möglichen Synergien seitens der Dozierenden und der Studierenden beantwortet werden.

Das auf dargestellter Grundlage und den entsprechenden Parametern motivierte Modell transmedialer Vermittlung zielt darauf ab, Fotografiegeschichte als wichtiges Element visue ller Kompetenz effizient im Sinne einer nachhaltigen und offenen Lehrveranstaltung greifbar zu machen. Dieses Konzept arbeitet sowohl mit Medien, die den Studierenden vertraut sind, also dem Computer und der Arbeit mit und im Internet, in Foren, Blogs und Bilddatenbanken, als auch mit weniger Vertrautem wie Fachliteratur, Quellenmaterial und Zeitschriftenartikel. Der Umgang ist dabei stets offen, spielerisch, fordernd, entdeckend und proaktiv. Das Modell zeigt eine mögliche Form der Anwendung exemplarisch. Es ist individuell und entsprechend dem Handlungskontext anzupassen und basiert auf einer Synthese von zwei unterschiedlichen Bereichen. Erstens gehören dazu Studien zur Effizienz und Wirksamkeit des Lehrens und Lernens, die im Hinblick auf das Unterrichten an Fachhochschulen befragt werden. Wesentliche Inspiration lieferte zweitens die

8 Hervorhebung im Original.

9 Der Autorin ist durchaus bewusst, dass es sich hierbei um eine verallgemeinernde Aussage handelt und dass es sowohl Studierende als auch Dozierende mit Lernkompetenz bzw. mit pädagogischer Ausbildung gibt. 
in einem ganz anderen Kontext situierte Forschung zu Computerspielen (educational games) und der Adaption dort verwendeter Begriffe wie "Cooperation», «Competition», «Achievement» und «Immersion» (Radoff 2011, 89-91; Rodriguez, Chengu. Basu 2010; Zheng 2009). Jon Radoff hat in seinem Buch zu «energize your business with social media games» aufgerufen und motiviert, einige seiner Überlegungen in das Modell transmedialer Vermittlung zu integrieren. Aus ersterem ergibt sich, das Bildinterpretation und Bildverstehen (Marotzki u. Niesyto 2006) integrative Bestandteile dieses Modells sind. Aus der Verbindung mit dem zweiten Bereich ist das Modell als innovatives Handwerkszeug (Hattie 2009, 24) zur Ausbildung visueller Kompetenz zu verstehen.

\section{Ein Modell transmedialer Vermittlung}

Die heutige Bilderflut ist ein zentrales Thema, das sich als Einstieg in die Fotografiegeschichte besonders eignet. Es geht um den Versuch, aus einem vermeintlich bildlichen und inhaltlichen Chaos heraus eine Ordnung und Übersicht zu schaffen, aus einem Gefühl der Ohnmacht gegenüber der Fülle und der Unübersichtlichkeit des historischen Materials mit bewussten Schritten ein Gefühl der Handhabung eben dieser Geschichte zu entwickeln. Der nachfolgend ausführlich dargestellte Einstieg verdeutlicht das auf Offenheit angelegte Modell. Diese methodologische und inhaltliche Offenheit soll unmittelbar ab dem ersten Moment des Zusammentreffens für Studierende transparent sein.

Über einhundert Abbildungen aus der gesamten Geschichte der Fotografie und auch der Kunst sind ohne Hinweis auf Künstlerin oder Künstler sowie einer Jahresangabe, in loser Folge, schwarzweiss und farbig, in verschiedenen Formaten, mit unterschiedlichen Sujets und einer möglichst grossen Breite eingesetzter Techniken zum ersten Sichten auszulegen. Die Studierenden sollen anschliessend die Bilder gemeinsam in eine Ordnung bringen. Die Aufgabe für die Gruppe lautet, dies zu tun, ohne sich zunächst verbal darüber zu verständigen. Ferner wird das Kriterium der Ordnung nicht expliziert. Erst wenn den Studierenden das Ziel der Lektion klar ist (Hattie 2009, 125-126; Helmke 2009, 85-86; Radoff 2011, 83), also vom Chaos zur Ordnung, von einer Bilderflut zu einer Bilderübersicht, kann ohne sprachliche Kommunikation und trotz einer gewissen Unklarheit hinsichtlich des Arbeitsauftrags gearbeitet werden. Es kann vorab durchaus kommuniziert werden, dass der Grund für den Verzicht auf Sprache und ein vorgegebenes Ordnungsprinzip in der Konzentration auf sinnliche, nonverbale Reize liegt, in dem auf Intersubjektivität angelegten Appell einer «Resonanz oder Korrespondenz» (Sowa u. Uhlig 2006, 78; Didi-Huberman, 2000). Und selbstverständlich ist es möglich, bei zu grosser Irritation ein entsprechendes Ordnungsprinzip zu nennen.

Wie kann man sich nun diesen Einstieg genauer vorstellen? Eine erste Abbildung freier Wahl wird von einer Studentin oder einem Studenten stillschweigend an die 
Wand geheftet. Alle anderen Studierenden hängen anschliessend nach ganz eigenen Kriterien und Ordnungsprinzipien weitere Abbildungen unmittelbar daneben oder auch weiter entfernt von ihr auf. Ebenso ist es erlaubt, auf die bisherige Ordnung keine Rücksicht zu nehmen und seinerseits einen neuen Bereich zu eröffnen. Die Studierenden übernehmen den aktiven Part an der Gestaltung des Unterrichts und werden zu Vermittlern ihres Wissens und ihrer Ansichten, während die Dozentin oder der Dozent Beobachter zweiter Ordnung ist (Hattie 2009, 25; Huber 2004b, 31). Die von den Studierenden kreierte Bilderwand wird dann später einen Mix von unterschiedlichen Ordnungsprinzipien beinhalten (Abb. 1). Unabhängig davon können die Studierenden daran ihre Kenntnisse von aktuellem und «traditionellem Bildreservoir» (Bering 2002, 91) sowohl auf spielerische als auch auf komparatistische Art auf die Bilderwand übertragen. Ferner wird das Sehen und das genaue Beobachten geschult, denn die Studierenden greifen immer wieder in den Prozess der Auswahl ein. Die reflektierende Perzeption gehängter Bilder fördert die Wahrnehmung und indirekt auch das Nachdenken über das eigene und über das fremde Handeln. (Breidbach 2005, 17-18; Hattie 2009, 173-178; Sowa u. Uhlig 2006, 78-79).

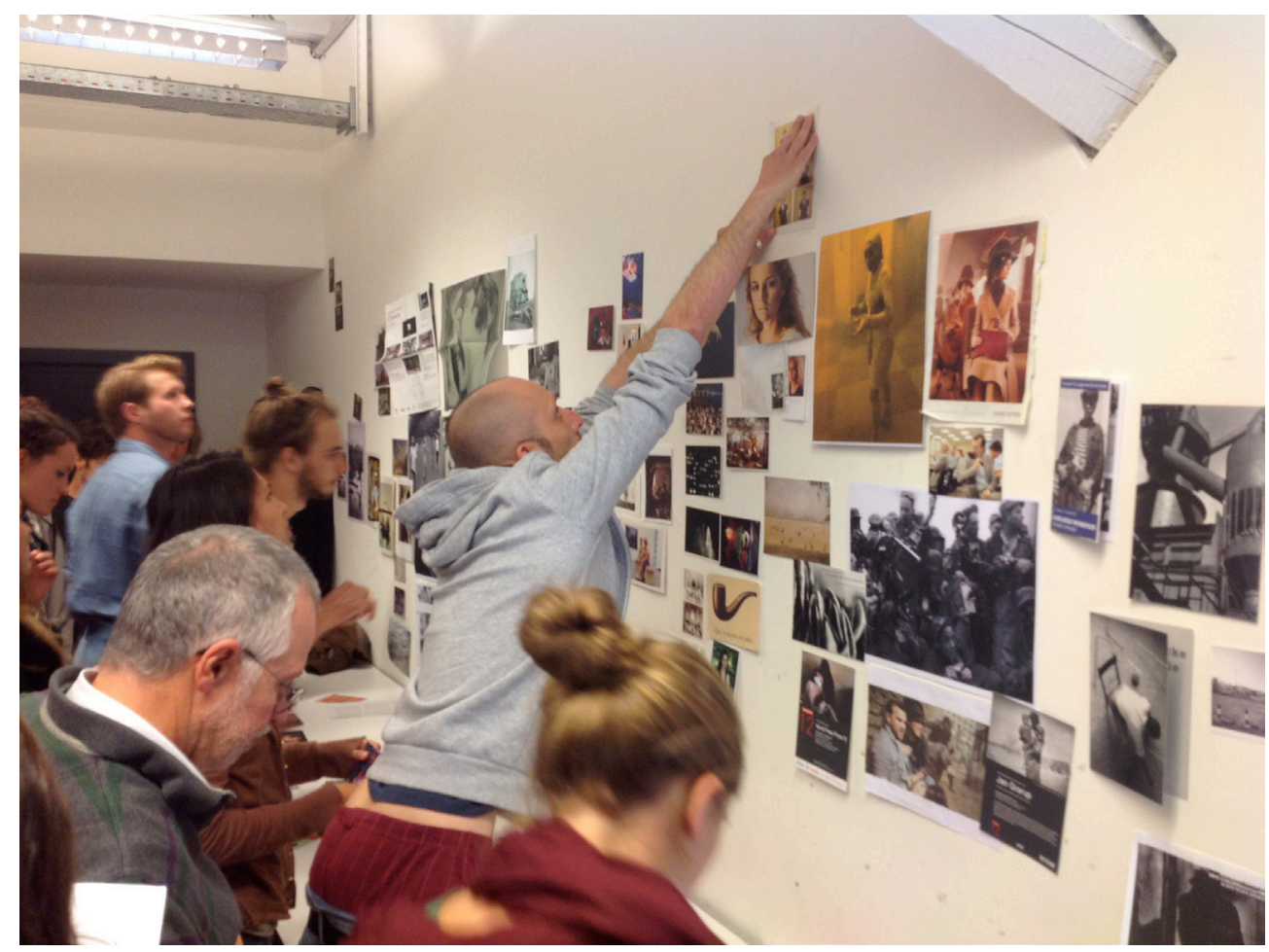

Abb. 1: Hochschule Luzern, Camera Arts, 2012. 
Nachdem alle Bilder an die Wand geheftet worden sind, werden die Studierenden gebeten, fünf Minuten weiterhin schweigend das Ergebnis kritisch zu betrachten. Die dozierende Person achtet auf die Einhaltung der Zeit. Anschliessend haben die Studierenden weitere fünf Minuten die Möglichkeit, bereits gehängte Bilder ohne Kommentar neu zu platzieren. Wichtig ist jeweils die Rückmeldung von den Studierenden an die dozierende Person, ob die Aufgabe verstanden wurde und ob klar ist, dass alle innerhalb des gegebenen Zeitfensters immer wieder in den Prozess der Ordnens, durchaus als eine Form von «social competition» (Radoff 2011, 83), eingreifen dürfen. Das Verständnis dient zum Gelingen der Aufgabe, aber primär geht es darum, den Studierenden die Verantwortung für ihr eigenes Handeln als Teil einer kollektiven Lehr- und Lerngruppe zu geben. Die dozierende Person ist Teil der Gruppe und übernimmt bis zu diesem Zeitpunkt drei Aufgaben: die Moderation der Aufgabenstellung, die Einhaltung der Zeitfenster und die genaue Beobachtung der Aktivitäten der Gruppe wie Einzelner (Hattie 2009, 238). Dafür sind Notizen und Fotografien zu machen, um das Handeln der Teilnehmenden sowie die einzelnen Stadien der entstehenden und der sich verändernden Bilderwand festzuhalten. Beides wird an anderer Stelle des Unterrichts erneut zu seinem Gegenstand gemacht; die Fotos und Notizen dienen insbesondere der Rückmeldung über gewonnene Erkenntnisse im Hinblick auf visueller Kompetenz. Konkret wird dies über die Implementierung der Fotografien im Intranet realisiert, zu denen Studierende als aktive Nutzer Zugang haben. Kommentare, Ergänzungen, eigene Fotografien können so beigestellt und abermals diskutiert werden. Ebenso kann von Studierenden ein Blog eingerichtet werden oder auch eine facebook-Seite, so dass auch Kolleginnen und Kollegen zugreifen und kommentieren können. Die Pflege der Seiten und das Bloggen selbst ist nach Absprache wechselseitig von allen Studierenden zu übernehmen (Porombka 2012). Unterschiedliche Ansichten befruchten die Diskussionen, Sprach- und Schreibkompetenz wird geübt und Wissen generiert. Über die sozialen Medien erhalten Inhalte auch Impulse von anderen Interessierten, die das im Unterricht Behandelte ergänzen und bereichern (Schulze 2003). Die klassische Rollenverteilung von Lehrperson einerseits und Lernenden andererseits ist aufgehoben, Synergien werden genutzt, das Wertegefühl der einzelnen Studierenden ist durch die Einbeziehung ihrer jeweiligen inhaltlichen, technologischen, medialen, u. a.m. Fähigkeiten gesteigert und lädt massgeblich zum Eintauchen in das Modell transmedialer Vermittlung ein und trägt somit auch zur Leistungssteigerung bei (Hattie 2009, 235-247; Radoff 2011). Nach dieser nonverbalen Arbeit der Gruppe wird nun das Ergebnis der Bilderwand im Plenum diskutiert. Es ist wichtig, alle Aussagen zuzulassen, Vermutungen über Fotografinnen und Fotografen, über Zeiteinteilungen, über etwaige andere Zusammenhänge, aber auch Gründe für Hängungen zu erforschen, offen für alle Formen von Überlegungen und Anregungen zu sein. Jede Aussage ist ernst zu 
nehmen und sollte zudem memoriert oder auch notiert werden. Über diesen intensiven Austausch kann erstens am Meinungsbildungsprozess des Gegenübers teilgenommen und zweitens durch das Plenum Wissen erfragt und vermittelt werden. Immer wieder kann erneut und kommentierend in die Bilderwand eingegriffen werden (Abb. 2). Die dozierende Person sollte dabei die Studierenden stets motivieren, ganz subjektiv ihre Meinungen zu formulieren. Dies betrifft auch die Klarheit und Umsetzbarkeit der Aufgabenstellung; das ist ein Aspekt, der Hatties Studie zu Folge wesentlich den Erfolg des Lernens bestimmt (2009, 125-126, 244-247). Gerade dieses proaktive Verhalten schafft die Basis des Vertrauens und der Gewissheit über ein positives Arbeitsklima, in dem scheinbare Fehler, Missverständnisse und Unkenntnis als Lernchance begriffen werden (Hattie 2009, 239). Die diachrone Ordnung der Bilder eröffnet, wie deutlich zu erkennen ist, verschiedene Möglichkeiten der Gruppierung. So wurden beispielsweise der Abbildung einer zentralperspektivisch aufgebauten Tafel von Martin Schaffner aus dem 16. Jahrhundert die Architekturfotografien von Charles Sheeler, 1927, Bernd und Hilla Becher aus dem Jahr 1972 und Yohiko Seino, 2001, sowie ein Poster der 2011 auf der Biennale Venedig gezeigten Installation von Christian Boltanski beigestellt.

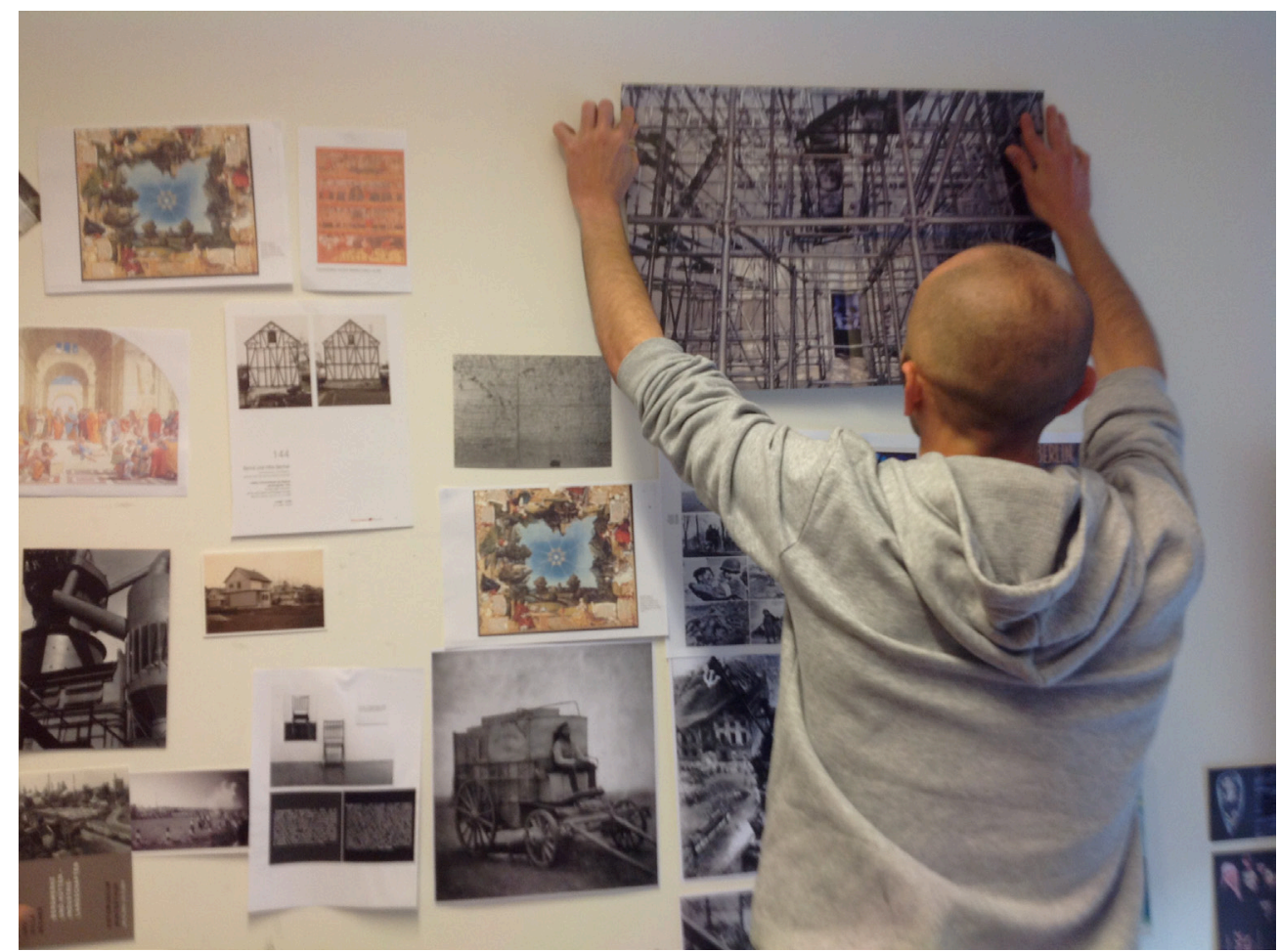

Abb. 2: Hochschule Luzern, Camera Arts, 2012. 
Als Ordnungsprinzip gaben die Studierenden die innerbildliche Betonung der Linien und Flächen an, woraufhin eine Studentin eine Abbildung der Fotografie von Henri Cartier-Bresson von Prostituierten in Alicante, 1933, dazu hing. Sie rechtfertigte ihr Handeln mit den Linien, die die liegenden Frauenkörper bilden würden, was wiederum andere Studierende motiviert hat, die nach Sujet, Grösse, Farbe, Technik oder Alter zusammen gestellten Bildergruppen aufzuheben und umzusortieren. Dieses Arbeiten mit individuellen Ordnungskriterien führte bei Studierenden allerdings recht schnell zur Unzufriedenheit und zum Wunsch, ein Kriterium zu benennen, das mit der Diversität der Abbildungen zu vereinbaren ist.

Bis zu diesem Zeitpunkt ist die dozierende Person primär Beobachter zweiter Ordnung und durch den Wechsel von Zurückhaltung und Präsenz in der Lage, aus der Perspektive der Studierenden die gesamte Lehr- und Lernsituation zu erfassen. Erkennen Studierende die Aufhebung der klassischen Rollenzuweisung, zeigt die Erfahrung, dass sie verstärkt ihre Kenntnisse und Anregungen Kommilitoninnen und Kommilitonen sowie Dozierenden mitteilen. An dieser Stelle des Unterrichtes ist von der dozierenden Person das Kriterium einer chronologischen Ordnung gemäss eines Zeitstrahls, oder für Studierende attraktiver formuliert, einer «timeline» zu diskutieren. Die Dozentin oder der Dozent moderiert nur so weit, als dass das Gesagte gegebenenfalls aufgegriffen und hervorgehoben wird, um es in den Kontext der kulturpolitischen, wissenschaftsgeschichtlichen und technischen Geschichte zu stellen. Angesichts der inhaltlichen Breite und interdisziplinären Verknüpfungen der Fotografie- und Kunstgeschichte wird deutlich, dass eine historische Erfassung der Geschichte für die Ausbildung einer visuellen Kompetenz Vorteile hat. Die danach folgende Bereitschaft an der aktiven Teilnahme am fotografiehistorischen Unterricht ist, so haben es die Unterrichtseinheiten gezeigt, eine vollkommen andere.

\section{Gemeinsam und für alle sichtbar Lernen}

Das aufgezeigte Modell eines spielerischen und komparatistischen Zugangs mit Betonung einer stärkeren Partizipation der Studierenden dient nicht nur dem sinnlichen Erleben von Bildmaterial und Geschichte. Es wird auch als Vereinbarung zu einer offenen Lernumgebung für alle Teilnehmenden verstanden. Im Verlauf der Unterrichtseinheit wird in regelmässigen Abständen mit einer chronologisch organisierten «timeline» in Papierform gearbeitet. Zwar gibt es mehrere Anbieter für elektronische «timelines», aber das hier vorzustellende Modell sieht gerade im haptischen Umgang mit der an die Wand zu heftenden Zeitleiste und den daran ebenfalls in Papierform anzubringenden Bildern und Zetteln einen besonderen Reiz, den die heutigen Studierenden als mehrheitlich «digital natives» sehr schätzen. Die dozierende Person kann den Aufbau der «timeline» zum Bestandteil des Unterrichts machen und sie mit den Studierenden gemeinsam herstellen. Andern- 
falls ist sie von der Dozentin oder dem Dozenten vorzubereiten und zu den ausgewählten Stunden an die Wand zu heften.

Das Bild- und Informationsmaterial sollte zum überwiegenden Teil von den Studierenden und nur ergänzend von der dozierenden Person mitgebracht werden. Lehrende sind Lernende und umgekehrt und profitieren von den unterschiedlichen Wahrnehmungen von Fotografie, Kunst und Medien. "When teachers see learning through the eyes of the student and when students see themselves as their own teachers» (Hattie 2009, 238), wird Lernen zu einem gemeinsamen und für alle sichtbaren Prozess. Wichtig ist sicherzustellen, dass ausreichend Material für den gewählten thematischen Schwerpunkt innerhalb des fotografiehistorischen Unterrichts bereit liegt. Die dozierende Person kann je nach Rücklauf der Bilder und Informationen sowie nach Rückmeldungen der Studierenden eher gezielte oder eher offene Hinweise und Aufträge dazu geben. Es wäre etwa zu gewährleisten, dass beispielsweise zum Thema "Bewegung» sowohl Aufnahmen von Bewegungsunschärfe aus den 1840er Jahren vorliegen als auch solche, die ganz aktuell den Kunstmarkt erobern, mit Preisen ausgezeichnet oder in der Presse diskutiert werden. Anhand des Materials ist die spezifisch fachliche Ebene, von der eingesetzten Technik bis zur Bilddeutung, von der Distribution bis zur Rezeption in der Breite des gegebenen Materials zu thematisieren. Anschliessend folgt bei jedem einzelnen Bild die Verortung auf der «timeline». Es werden die historische Situierung der Aufnahme und ihre Beziehung zu entsprechenden kunst- und kulturpolitischen Ereignissen in der Geschichte augenscheinlich, wenn dies zusätzlich zu einer mündlichen Thematisierung visuell auf der «timeline» markiert wird. Hierfür sind im Laufe des Semesters auf besonders gestaltetem Papier, also beispielsweise auf farbigem Karton in Form von Dreiecken, Sprechblasen, Sternen oder Kreisen, stichwortartige Hinweise zu ausgesuchten Momenten aus Geschichte und Gegenwart zu notieren und als so genannte Spots auf der «timeline» anzubringen (Abb. 3). Diese Momente können Kriege, Krisen, Katastrophen sein, politische Umbrüche, Gesellschaftswandel, Mode, globale Ereignisse, wichtige theoretische Abhandlungen u. v. m, ebenso technische Begriffe, Zeitfenster mit den Bezeichnungen für künstlerische Bewegungen, Namen wichtiger Vertreterinnen und Vertreter der einzelnen «Ismen», Institutionen und Personen, Zitate von Politikern, wichtige Ausstellungen, u. s. w. Die «Verschlagwortung» des Unterrichts wird also auch unabhängig von der Arbeit mit der «timeline» durchgeführt; sie dient aber in den Stunden vor der chronologisch organisierten Bilderwand ganz dezidiert zur Reflexion von Inhalten, zur oben genannten Sichtbarmachung von Bezügen und Zusammenhängen sowie zur Rückmeldung an die dozierende Person über Erkenntnisse, mögliche Fehlstellen, Umsetzung und Durchführbarkeit von gestellten Aufgaben (Hattie 2009, 125-126, 244-247). 


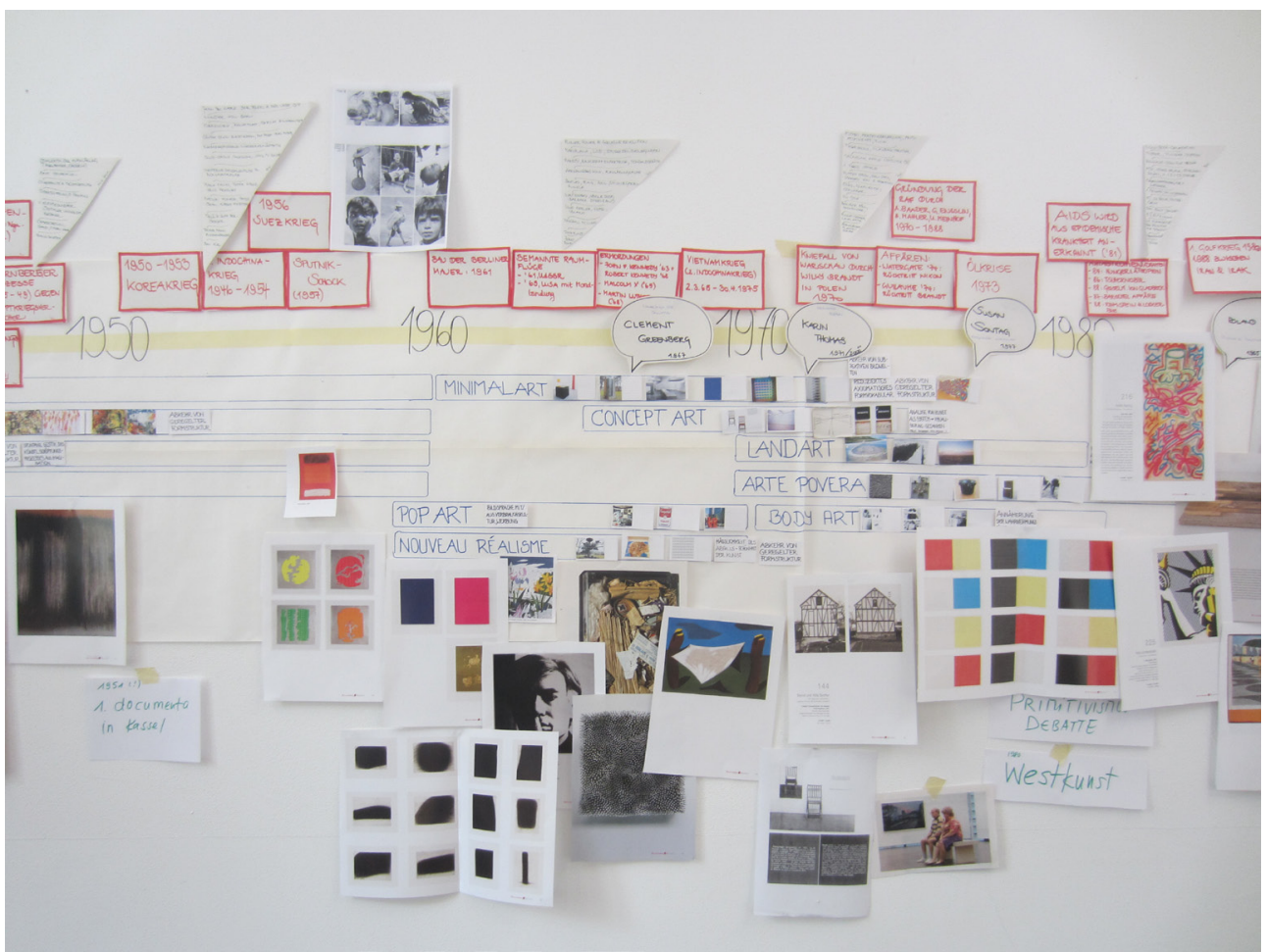

Abb. 3: Zürcher Hochschule der Künste, Pooling Theorie, 2011.

Methodisch sind für die Erschliessung dieser zusätzlichen Bereiche und ihrer nachfolgenden Übertragung in einen «Spot» verschiedene Möglichkeiten vom Einzelauftrag bis zur gezielten Gruppenarbeit denkbar. Die Studierenden üben darüber die Fähigkeit ein, zu einem Thema nach Literatur zu forschen, das Internet nach verbürgten Informationen zu befragten und verständliche Aussagen, Texte sowie Exzerpte und Referate für die Gruppe bereit zu halten. Mitunter werden die danach folgenden Referate und Präsentationen kaum neues Material bereit stellen; allerdings machen sich die Studierenden in der Regel gegenseitig auf die unzureichende Erledigung der Aufgabe aufmerksam und fordern zur Nachbesserung auf. Die Anbringung und Präsentation der Ergebnisse an der «timeline» können durchaus Wettbewerbscharakter haben. Das Plenum ist dazu aufgefordert, die Rolle einer Jury einzunehmen, um sich über den Inhalt, die Rhetorik und die eingesetzten Mittel zu verständigen. Diese Integration einer Rückmeldung durch Ihresgleichen in einer wettbewerbsähnlichen Situation fördert und fordert auf beiden Seiten die kognitive, sprachliche und reflexive Kompetenz und das Engagement der Teilnahme (Huber 2004a; Radoff 2011). Durch das Einnehmen einer Rolle sind auch weniger aktive Studierende eingeladen, vollumfänglich Stellung beziehen zu müssen. Von ihnen wird hierbei in hohem Mass gefordert, ihre visuelle Kompetenz ein- 
zusetzen, um ernstzunehmende und konstruktive Kritik verbalisieren zu können. Interessanterweise sehen die zu bewertenden Studierenden in der vermeintlichen Jury in der Regel primär ihre Studienkolleginnen und -kollegen und wehren sich erfahrungsgemäss wesentlich stärker gegen Kritiken der Dozierenden. Für diese ist dies wiederum ein besonders erhellender Moment.

Bei dieser ergänzenden Arbeit fliesst auch die Lektüre von Texten ein, durch die gezielt Inhalte erschlossen werden können, die dann ihrerseits als "Spots» die «timeline» ergänzen. Zunächst aber muss das Lesen und das Verfassen von Exzerpten eingeübt werden. Hierbei hat die dozierende Person Vorbildfunktion, in dem sie aus der Situation heraus und unter Mithilfe der Studierenden ein Exzerpt verfasst. Dies ist zwingend mit und vor den Studierenden auf ein White- oder Blackboard, der Folie eines Hellraumprojektors oder direkt in ein Dokument bzw. auf der Folie einer Power Point Präsentation zu verschriftlichen. Das Geschriebene ist somit für alle sichtbar, veränderbar und zu ergänzen. Bei der Analyse mehrerer Texte sollte ebenso offen verfahren werden und dabei die auf Schlagworte reduzierten Aussagen der Studierenden aufgenommen werden. Mittels Verbindungslinien wird vor Augen geführt, dass verschiedene Autorinnen und Autoren aus ihrer jeweiligen Perspektive letztlich trotz historisch oder theoretisch weit auseinander liegenden Positionen über ähnliche oder gar dieselben Dinge reden. Es hat sich gezeigt, dass die Reduktion auf Wesentliches die Studierenden im Umgang mit wissenschaftlichen Texten deutlich entspannt und ihnen den Zugang zu derartigen Texten im Nachfolgenden erleichtert hat. Nur über dieses Handeln wird es zu einem späteren Zeitpunkt gelingen, dies auch von den Studierenden zu verlangen und später von Sitzung zu Sitzung Protokolle anfertigen zu lassen. Texte als externe Repräsentationsformen von Wissen werden hier in eine Darstellung der Re-Präsentation überführt und potenzieren damit in dieser anschaulichen Weise die von Wolfgang Schnotz konstatierte «perzeptuelle Repräsentation, auf deren Grundlage ein mentales Modell des abgebildeten [und des dargestellten, M. G.] Sachverhalts konstruiert wird» $(2003,26$.). Fotografien der ephemeren Ergebnisse oder digitale Scripte ergänzen die Einträge im Intranet beziehungsweise im Internet und bilden so einen weiteren Zweig transmedialer Vermittlung.

Eine ausgesprochen lustvolle, zugleich aber auch herausfordernde Methode bei der Diskussion von Textinhalten und der Reflexion über die Haltung ausgewählter Autorinnen und Autoren stellt das inszenierte Podiumsgespräch dar. Die Studierenden schlüpfen dazu in die Rollen von Textautorinnen und -autoren. Sie sind aufgefordert, in einem moderierten und zeitlich begrenzten Gespräch den jeweiligen methodischen Ansatz der von ihnen ausgewählten Autoren zu formulieren, ebenso wie Hauptthesen und Inhalte. Die Namen der so behandelten Autorinnen und Autoren sowie ihre wichtigsten Themen, Thesen oder auch Aussagen können abermals als «Spot» die «timeline» ergänzen. Die Vorbereitung zum Gespräch verläuft 


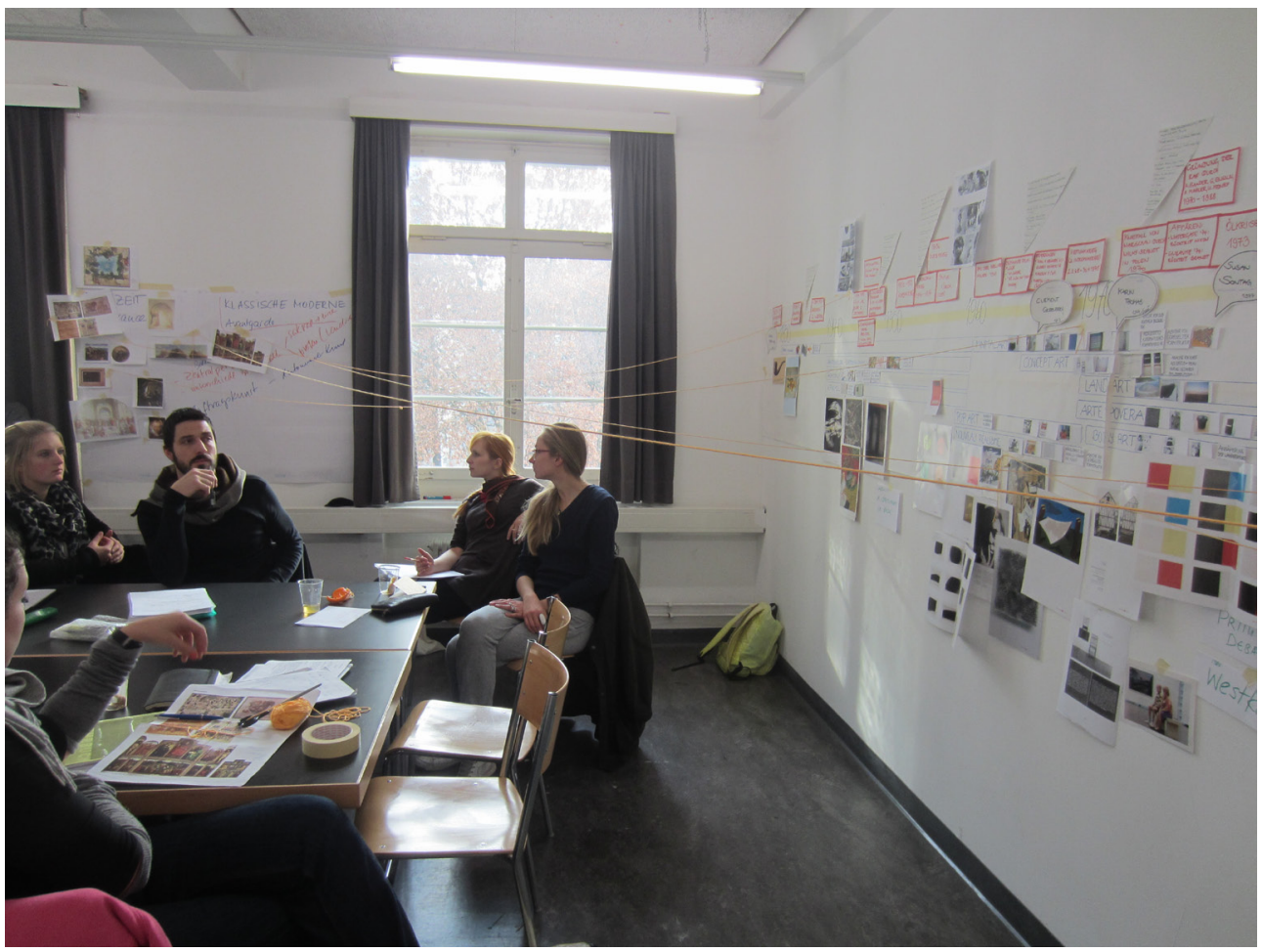

Abb. 4: Zürcher Hochschule der Künste, Pooling Theorie, 2011.

in Kleingruppen (Schnotz 2006), aus denen dann eine Podiumsteilnehmerin oder ein Podiumsteilnehmer ausgewählt wird. Das Gespräch wird vom Plenum verfolgt, das am Ende aufgefordert ist, selbst Fragen zu formulieren. Eine filmische oder fotografische Dokumentation im Intranet oder Internet dient der Fortführung, Erweiterung, Ergänzung und Kommentierung der Inhalte und so zu einer referentiellen Verknüpfung verschiedener Ebenen miteinander (Mayer 2005). Mittels «Ausschöpfung mehrerer Sinneskanäle und Erlebnismodalitäten, der Anreicherung durch Verknüpfungen zu Alltagsphänomenen sowie durch konkrete Sinneserfahrungen» (Helmke 2009, 62) wird das Ziel einer «breiten, vielfältigen Verankerung im Langzeitgedächtnis» verfolgt.

Die Arbeit mit und an der sich permanent erweiternden «timeline» übt den Reiz aus, sie so umfassend wie möglich zu ergänzen. Es ist vorgekommen, dass Studierende in den Pausen bereits bestehende Bilder und "Spots» vor der "timeline» diskutiert und die noch vorhandenen Lücken mit Hinweisen, konkreten Namen u. a.m. gefüllt haben. Ebenso haben Gruppen von zwei oder drei Personen ohne Aufforderung begonnen, selbstständig die Abbildungen auf der «timeline» zu verorten. Ab einem bestimmten Moment der deutlich gefüllten «timeline» wurden Fragen nach etwaigen Verbindungen gestellt, die technischer, ästhetischer, inhalt- 
licher oder auch kulturpolitischer Art waren. Und dieses Herstellen von Verbindungen ist es, was daran ab der Mitte, auf jeden Fall aber am Ende des Semesters deutlich zu machen ist.

Die vielschichtigen Möglichkeiten, die sich während der Arbeit an der «timeline» eröffnet haben, werden durch ihre explizite Linearität räumlich beschränkt. Die «timeline» bleibt ein Modell des zeitlichen Vor- und Nacheinanders. Um dennoch über die Linearität eines Zeitstrahls hinaus Zusammenhänge und Verbindungen inhaltlich und visuell erweitert kenntlich zu machen, sind an Abbildungen und «Spots» der «timeline» farbige Fäden mit Haftklebern festzumachen und durch den Raum zu einem anderen Bereich auf der «timeline» zu ziehen. Damit kann der linearen Einschränkung entgegen gewirkt und können divergente Momente räumlich verbunden werden (Abb. 4). Diese Handlungen können von allen Beteiligten vorgenommen werden. Die dozierende Person übernimmt dabei zumindest den Hauptpart der Moderation. Die Studierenden ergänzen die Verbindungen, geben neue Hinweise, formulieren andere Sichtweisen, kommentieren und kritisieren. In dieser abschliessenden, raumgreifenden Performativität zeigt sich für die Studierenden in aller Deutlichkeit ihre intersubjektive visuelle Kompetenz. Werden nun zusätzlich die von der dozierenden Person gemachten dokumentierenden Fotografien der ersten Lektionen projiziert, auf denen die noch wenig fassbaren Ergebnissen an den Wänden, die ersten Kommentare in den Blogs oder im Intranet zu sehen sind, werden sich die Studierenden selbst-bewusst, dass sich ihre visuelle Kompetenz erweitert hat, und dass Fotografiegeschichte durchaus gestaltbar, erweiterbar und greifbar ist. 


\section{Bibliografie}

Adorno, Theodor. 1970. Erziehung zur Mündigkeit. Frankfurt/M.: Suhrkamp.

Bering, Kunibert. 2002. "Bezugsfelder der Vermittlung visueller Kompetenz.» In Bild, Medien, Wissen. Visuelle Kompetenz im Medienzeitalter, hrsg. v. Hans Dieter Huber, Bettina Lockemann u. Michael Scheibel, 89-101. München: kopaed.

Breidbach, Olaf. 2005. Bilder des Wissens: Zur Kulturgeschichte der wissenschaftlichen Wahrnehmung. München: Fink.

Didi-Huberman, Georges. 2000. Vor einem Bild. (frz. Originalausg. 1990). München u. Wien: Carl Hanser.

Ganguin, Sonja und Dorothee Meister, Hrsg. 2012. Digital native oder digital naiv? München: kopaed.

Habermas, Jürgen. 2004/1962. Strukturwandel der Öffentlichkeit: Untersuchungen zu einer Kategorie der bürgerlichen Gesellschaft. Unverändeter Nachdr. der 9. Aufl. mit Vorw. zur Neuaufl. 1990. Frankfurt am Main: Suhrkamp.

Hattie, John. 2009. Visible Learning: A synthesis of over 800 meta-analyses relating to achievement. London u. New York: Routledge.

Helmke, Andreas. 2009. Unterrichtsqualität und Lehrerprofessionalität: Diagnose, Evaluation und Verbesserung des Unterrichts. 2., aktualisierte Aufl. Seelze-Velber: Klett Kallmeyer.

Huber, Hans Dieter. 2004a. «Im Dschungel der Kompetenzen.» In Bild, Medien, Wissen: Visuelle Kompetenz im Medienzeitalter, hrsg. v. Hans Dieter Huber, Bettina Lockemann u. Michael Scheibel, 15-29. München: kopaed.

Huber, Hans Dieter. 2004b. "Visuelle Performativität.» In Bild, Medien, Wissen: Visuelle Kompetenz im Medienzeitalter, hrsg. v. Hans Dieter Huber, Bettina Lockemann u. Michael Scheibel, 31-37. München: kopaed.

Macho, Thomas. 1999. «lst mir bekannt, dass ich sehe?) Wittgensteins Frage nach dem inneren Sehen.» In Video ergo sum: Repräsentation nach innen und aussen zwischen Kunst- und Neurowissenschaften, hrsg. v. Olaf Breidbach u. Karl Clausberg, 99-111. Hamburg: Hans-Bedow-Institut.

Marotzki, Winfried und Horst Niesyto, Hrsg. 2006. Bildinterpretation und Bildverstehen: Methodische Ansätze aus sozialwissenschaftlicher, kunst- und medienpädagogischer Perspektive. Wiesbaden: VS Verlag für Sozialwissenschaften.

Mayer, Richard E. 2005. Multimedia Learning. 7. Aufl. Cambridge: Cambridge Press.

Merleau-Ponty, Marcel. 1966. Phänomenologie der Wahrnehmung. Berlin: W. de Gruyter.

Mitchell, William John Thomas. 1994. Picture Theory: Essays on verbal and visual representation. Chicago: The University of Chicago Press.

Porombka, Stephan. 2012. Schreiben unter Strom: Experimentieren mit Twitter, Blogs, Facebook \& Co. Mannheim u. Zürich: Duden. 
Posner, Roland. 2003. «Ebenen der Bildkompetenz.»In Was ist Bildkompetenz? Studien zur Bildwissenschaft, hrsg. v. Klaus Sachs-Hombach, 17-23. Wiesbaden: DUV.

Prensky, Marc. 2001. Digital Natives, Digital Immigrants, On the Horizon (MCB University Press), Vol. 9 No. 5.: http://www.marcprensky.com/writing/prensky\%20 -\%20digital\%20natives,\%20digital\%20immigrants\%20-\%20part1.pdf.

Radoff, Jon. 2011. Game on: Energize Your Business with Social Media Games. Indianapolis: Wiley Publishing.

Rodriguez, Saul, Irene Cheng und Anup Basu. 2010. «Educational Games.» In Multimedia in Education: Adaptive Learning and Texting, hrsg. v. Lluís Vicent Safont, Anup Basu u. Rand Goebel, 77-124. New Jersey: Word Scientific.

Sachs-Hombach, Klaus, Hrsg. 2003. Was ist Bildkompetenz? Studien zur Bildwissenschaft. Wiesbaden: DUV.

Schaper, Florian. 2012. Bildkompetenz: Kunstvermittlung im Spannungsfeld analoger und digitaler Bilder. Bielefeld: transcript.

Schnotz, Wolfgang. 2006. "Bild- und Sprachverarbeitung aus psychologischer Sicht.» In Was ist Bildkompetenz? Studien zur Bildwissenschaft, hrsg. v. Klaus Sachs-Hombach, 25-42. Wiesbaden: DUV.

Schulze, Gerhard. 2003. Die beste aller Welten: Wohin bewegt sich die Gesellschaft im 21. Jahrhundert? München: Hanser.

Sowa, Hubert und Bettina Uhlig. 2006. «Bildhandlungen und ihr Sinn: Methodenfragen einer kunstpädagogischen Hermeneutik.» In Bildinterpretation und Bildverstehen: Methodische Ansätze aus sozialwissenschaftlicher, kunst- und medienpädagogischer Perspektive, hrsg. v. Winfried Marotzki u. Horst Niesyto, 77-106. Wiesbaden: VS Verlag für Sozialwissenschaften.

Weingarten, Michael. 1999. «Wahrnehmungen haben und Erfahrungen machen?» In Video ergo sum: Repräsentation nach innen und aussen zwischen Kunst- und Neurowissenschaften, hrsg. v. Olaf Breidbach u. Karl Clausberg, 158-180. Hamburg: Hans-Bedow-Institut.

Weinert, Franz Emanuel. 2009. "Sechs fundamentale Bildungsziele.» In Unterrichtsqualität und Lehrerprofessionalität: Diagnose, Evaluation und Verbesserung des Unterrichts, hrsg. v. Andreas Helmke, 46. 2., aktualisierte Aufl. SeelzeVelber: Klett Kallmeyer.

Zawacki-Richter, Olaf, Eva Maria Bäcker und Anke Hanft. 2010. «Denn wir wissen nicht, was sie tun ... Portfolios zur Dokumentation von Kompetenzen in einem weiterbildenden Masterstudiengang.» MedienPädagogik 18 (5. Feb.): http:// www.medienpaed.com/Documents/medienpaed/18/zawacki1002.pdf

Zheng, Robert. 2009. Cognitive Effects of Multimedia Learning. Hershey, New York: Information Science Reference. 